\title{
Vertebral Erosion After Chronic Rupture of an Infrarenal Abdominal Aortic Aneurysm: About One Case \\ Nya $F^{*}$, Atmani N, Seghrouchni A, Aithoussa M, and Boulahya A
}

Cardiac Surgery Department, Mohamed V Military Hospital, Faculty of Medicine, Mohamed V University, Rabat, Morocco

\section{Introduction}

Erosion of the lumbar vertebral body is a rare complication of a chronic rupture of the abdominal aorta aneurysm (AAA). Our study illustrates this complication through a clinical case and discusses the diagnosis and management a chronic rupture of the AAA.

\section{Case Report}

We report the observation of Mr K.M, 55 years old, former tobacco. He has been followed for one year due to chronic lower back pains with an episode of acute abdominal pain with posterior irradiation toward the lumbar fossae. These pains were initially treated as colic nephritis. The evolution is made towards the recurrence of pain. An abdominal ultrasound was performed during a painful crisis that showed intraabdominal effusion and the patient was referred to our training for management. Clinical examination at admission revealed arterial pressure of 100/50 $\mathrm{mmHg}$, cardiac frequency of $100 \mathrm{cycles} / \mathrm{min}$, and the abdomen was flexible but sensitive. The biological balance found hemoglobin levels of $7 \mathrm{~g} / \mathrm{dl}$. The abdominal CT showed a false aneurysm of the infrarenal abdominal aorta ruptured in the retroperitoneum and left psoas (Figures 1 and 2). The patient was emergently admitted to the operating room after the revelation of aortic clamping in a retroperitoneal hematoma. Surgical exploration found an infrarenal abdominal aortic aneurysm ruptured in the posterior and an Erosion of two lumbar vertebral bodies (L2 and L3). The hematoma fused to the Left iliac fossa.

Flattening of the aneurysm and interposition of an aorto-aortic tube in dacron. The postoperative progression was favorable and the patient left the hospital after 10 days of hospitalization.

\section{Discussion}

The chronic rupture of an aneurysm of the abdominal aorta is an

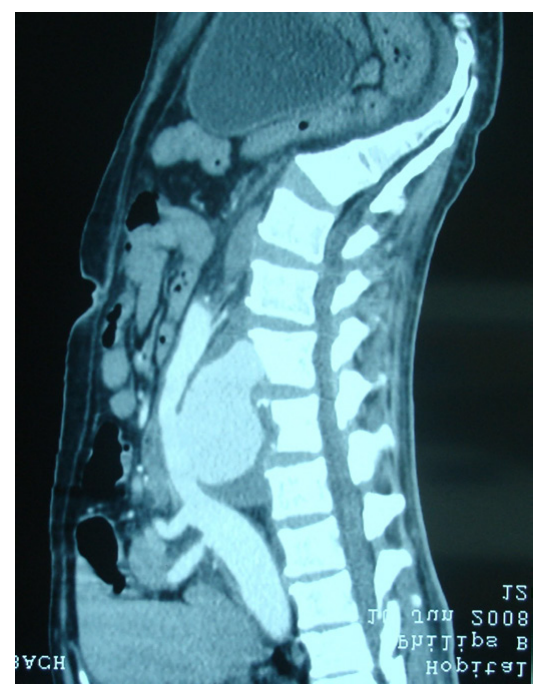

Figure 1: Scannographic image showing a ruptured $A A A$ in the retroperitoneum with erosion of the L2 and L3 vertebral bodies.

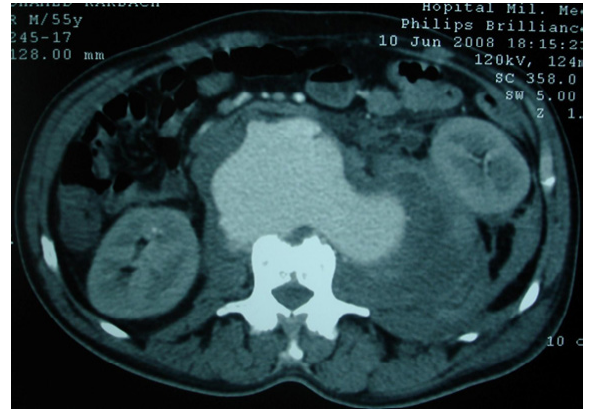

Figure 2: Scanned image showing a ruptured AAA fuse in the left psoas.

unusual event. The first published occurrence was in 1961 by Szilagyi [1]. Its frequency varies between 1.2 and $2.7 \%$ [2]. The peculiarity of these ruptures of aneurysms is their unusual symptomatology leading to its misdiagnosis. Chronic back or lumbar pain is most commonly noted. It may be accompanied by irradiation in a lower limb resembling a case of sciatica or cruralgia when the hematoma infiltrates the psoas muscle. The second misleading clinical feature is hemodynamic stability and the absence of signs of shock.

The diagnostic paraclinical examination of choice is computed tomography [3]. These ruptures are characterized by a density of the "soft tissue" type well differentiated with respect to the aorta, a discontinuity of the aortic circular calcifications with posterior defect and a masked psoas muscle [2]. The CT also makes it possible to demonstrate the erosion of a vertebral body which can as in our observation complicate this type of rupture. This complication may present differential diagnosis problems with vertebral lysis due to another mechanism [4]. Besides this rheumatological presentation, we must not disregard the pseudoseptic forms. A genuine infectious aneurysm may also become more complicated by chronic rupture and it was not uncommon in the past to observe this type of vertebral erosion in cases of syphilitic aneurysm [5].

These aneurysms must be operated in "semi-emergency". A clinical evaluation of patients and morphological aneurysms must nevertheless precede the surgery. Open surgery currently remaining the reference

*Corresponding author: Nya F, Cardiac Surgery Department, Mohamed V Military Hospital, Faculty of Medicine, Mohamed V University, Rabat, Morocco, E-mail: fouad.nya@hotmail.fr

Received February 13, 2017; Accepted March 13, 2017; Published March 20 , 2017

Citation: Nya F, Atmani N, Seghrouchni A, Aithoussa M, Boulahya A (2017) Vertebral Erosion After Chronic Rupture of an Infrarenal Abdominal Aortic Aneurysm: About One Case. J Cardiovasc Dis Diagn 5: 262. doi: 10.4172/23299517.1000262

Copyright: (C) 2017 Nya F, et al. This is an open-access article distributed under the terms of the Creative Commons Attribution License, which permits unrestricted use, distribution, and reproduction in any medium, provided the original author and source are credited. 
Citation: Nya F, Atmani N, Seghrouchni A, Aithoussa M, Boulahya A (2017) Vertebral Erosion After Chronic Rupture of an Infrarenal Abdominal Aortic Aneurysm: About One Case. J Cardiovasc Dis Diagn 5: 262. doi: 10.4172/2329-9517.1000262

treatment then makes it possible to obtain a morbidity-mortality comparable to that of the conventional regulated AAA surgery [5]. The endovascular treatment, whose technical feasibility has now been demonstrated for the treatment of broken AAA, seems also to be an interesting therapeutic alternative [6]. In cases with severe vertebral destruction, an osteosynthesis of the vertebral bodies may also be necessary [7].

\section{Conclusion}

The dorsal pain caused by chronic aneurysms of the abdominal aorta is a rare case and may be one of the possible aetiologies in a differential diagnosis of low back pain and/or chronic sciatica.

\section{References}

1. Szilagyi DE, Smith RF, Macksood AJ, Whitcomb JG (1961) Expanding and ruptured abdominal aortic aneurysms. Problems of diagnosis and treatment. Arch Surg 83: 395-408
2. Ando M, Igari T, Yokoyama $H$, Satokawa $H$ (2003) CT features of chronic contained rupture of an abdominal aortic aneurysm. Ann Thorac Cardiovasc Surg. 9: 274-278.

3. Ubukata H, Kasuga T, Motohashi G, Katano M, Tabuchi T (2005) Spinal destruction induced by chronic contained rupture of an abdominal aortic aneurysm: Report of a case. Surg Today 35: 411-414.

4. Higgins R, Peitzman AB, Reidy M, Stapczynski S, Steed DL, et al. (1988) Chronic contained rupture of an abdominal aortic aneurysm presenting as a lower extremity neuropathy. Ann Emerg Med 17: 284-287.

5. Sraieb T, Ben AY, Bougamra S, Longo S, Ben RN, et al. (2004) Chroniccontained ruptured aortic aneurysm: An unusual cause of back pain. Tunis Med 82: 1052-1055.

6. Seelig MH, Berchtold C, Jakob P, Schonleben K (2000) Contained rupture of an infrarenal abdominal aortic aneurysm treated by endoluminal repair. Eur $\mathrm{J}$ Vasc Endovasc Surg 19: 202-204

7. Aydogan M, Karatoprak O, Mirzanli C, Ozturk C, Tezer M, et al. (2008) Severe erosion of lumbar vertebral body because of a chronic ruptured abdominal aortic aneurysm. The Spine J 8: 394-396. 